\title{
Urine ketones in patients with acute appendicitis
}

Keywords: parameters, polymorphonuclear, neutrophilia, appendicitis, anorexia, nausea

\section{Introduction}

Acute appendicitis is the most common abdominal surgical emergency and its prognosis is dependent on accurate, early diagnosis. The diagnosis of acute appendicitis relies heavily on clinical judgment. ${ }^{1}$ No single aspect of clinical presentation can accurately predict the presence of the disease but a combination of various signs and symptoms may support the diagnosis. Unfortunately, acute appendicitis is still difficult to diagnose and misdiagnosis is not uncommon in the emergency department. In addition, unnecessary appendectomy can lead to postoperative complications, such as adhesion or infection. Furthermore, delay in accurate diagnosis may result in appendix perforation and its consequences. Therefore, improving accuracy is desirable both for earlier and accurate diagnosis. ${ }^{2}$ Different tests can be used to detect the presence of acute appendicitis. These tests include CDC, abdomen-pelvic ultrasound, CRP and sometimes abdomen pelvic CT, however none of these tests are neither $100 \%$ specific nor $100 \%$ sensitive. Many studies have been conducted to find out whether any other parameters can predict acute appendicitis. In some studies, equivocal urinalysis results have been reported. ${ }^{3}$

\section{Purpose}

In this study, we aimed to determine whether routine urinalysis may serve as a helpful tool in diagnosing acute appendicitis. The main purpose of the study is to determine if there is a correlation between acute appendicitis and ketones in the urine.

\section{Patients and methods}

The study was conducted in the Specialty Hospital in Amman, Jordan where clinically, acute appendicitis is often diagnosed based on correct history, taking physical examination and laboratory tests. A patient with clinical symptoms and signs which includes migration of pain, anorexia, nausea or emesis, tenderness in the RLQ of the abdomen, pyrexia, leukocytosis and polymorphonuclear neutrophilia and imaging such as abdomen-pelvic ultrasound or CT suggesting acute appendicitis is defined as suspected of having acute appendicitis. Most of the patients within the Specialty Hospital undergo routine urine analysis in the Emergency Room (ER) as part of investigation of the abdominal pain. Once diagnosis of acute appendicitis is made, emergent surgical approach is indicated. In this study, we reviewed the files of patients who have undergone appendectomy in the hospital from 31/12/2010 till 31/12/2015 and correlated them with the urine analysis findings.

\section{The inclusion criteria include}

i. Patients who have undergone appendectomy and have the histopathology report of the appendix.

ii. Patients who have also undergone routine urine analysis prior to the surgery.
Volume 4 Issue 6 - 2017

\author{
Abdullah Ramadan,' Ahmed Al Shneiwer, ${ }^{2}$ \\ Ahmed Al Nuirat ${ }^{2}$ \\ 'Surgical Resident, The Specialty Hospital, Jordan \\ ${ }^{2}$ General Surgery, The Specialty Hospital, Jordan
}

Correspondence: Abdullah Ramadan, Surgical Resident, The Specialty Hospital, 16 Abdel Qader Badran St Dabouq, Jordan, Tel 00962799993895, Email dr.a.ramadan@outlook.com

Received: January 17, 2017 | Published: August 17, 2017

\section{The exclusion criteria include}

i. Patient initially diagnosed as suspected acute appendicitis and not undergoing surgery.

ii. Absence of a histopathology report.

iii. Absence of routine urine analysis prior to surgery.

The number of patients admitted to the Specialty hospital diagnosed with acute appendicitis was 682 . The number of patients who have met the criteria of having a histopathology report of the appendix and a urine analysis prior to surgery was 303 . However, 2cases of these were excluded from the study as their histopathology report indicated the patients were diagnosed with carcinoid tumor. Furthermore, 1case was also excluded that showed an appendicular diverticulum. In conclusion, there were 300 cases used for the study. The patient group comprised 156 males (52\%) and 144 females (48\%); the mean age was 27.1years (range 5 to 82 years). Of these, 175 had histologically proven simple acute appendicitis, 11 had perforated and 73 had normal appendices.

\section{Statistical analysis}

The Chi-squared test was used and the results from patients with simple appendicitis were compared with those with non-inflamed appendix and also with perforated appendicitis. A p-value of less than 0.05 was considered statistically significant. The results showed a nonsignificant correlation between ketones in the urine in differentiating non-inflamed appendix from an inflamed appendix. However, it showed a significant correlation in differentiating early appendicitis from late gangrenous appendix (Table 1). Figure 1 illustrates that the highest number of cases (186) were those with inflamed appendix and tested negative for ketones in the urine, the lowest number of cases were those with non-inflamed appendix and tested positive for ketones in the urine (Table 2). Figure 2 illustrates that the highest number of cases (175) were those with early appendicitis and tested negative for ketones in the urine. The lowest number of cases (8) was those with perforated appendix and tested positive for ketones in the urine. 


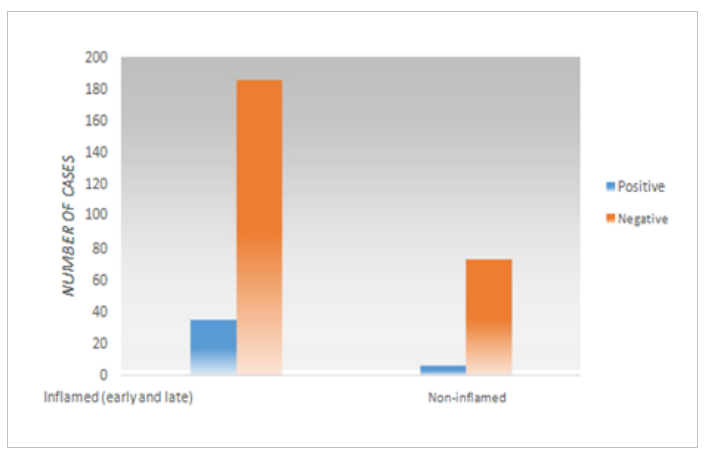

Figure I Comparison of the number of inflamed and non-inflamed appendix cases with positive and negative ketones.

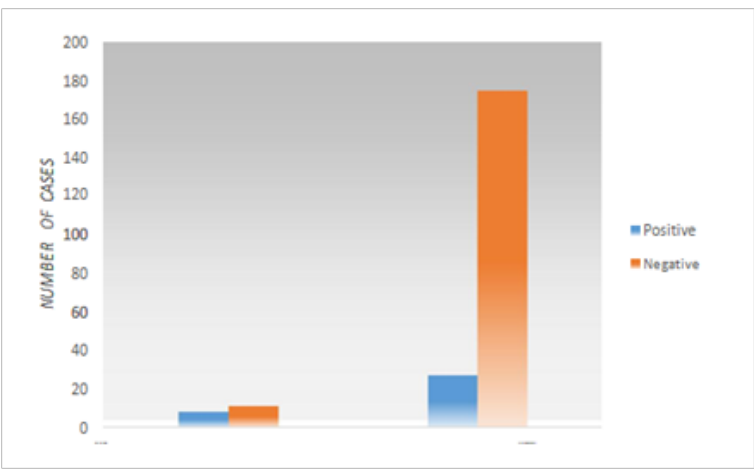

Figure 2 Comparison of the number of perforated and early appendicitis cases with positive and negative ketones.

Table I Classification of inflamed and non-inflamed cases with positive and negative ketones

\begin{tabular}{lll}
\hline Ketones & Inflamed (early and late) & Non-inflamed \\
\hline Positive & 35 & 6 \\
Negative & 186 & 73 \\
\hline
\end{tabular}

*The Chi-squared test indicated the $\mathrm{p}$ value was 0.07 .

Table 2 Classification of perforated and early appendicitis cases with positive and negative ketones

\begin{tabular}{lll}
\hline Ketones & Perforated & Early appendicitis \\
\hline Positive & 8 & 27 \\
Negative & 11 & 175 \\
\hline
\end{tabular}

*The Chi- squared test indicated the $\mathrm{p}$ value was $0.00 \mathrm{I}$.

\section{Discussion}

Acute appendicitis is often diagnosed based on correct history taking, physical examination and laboratory tests. On the basis of its low sensitivity and high specificity in the results, routine urinalysis may not serve as an effective tool in predicting acute appendicitis but may aid clinicians in decision making to rule out acute appendicitis and so lower negative appendectomy rates. ${ }^{4}$ In this study, significantly higher percentage of ketone bodies in patients with late appendicitis than in those with early appendicitis has been found. Nevertheless, there were some limitations in this study. Firstly, based on the results of the study, a higher specificity and a low sensitivity indicated that urine ketones may aid clinicians in ruling out perforated appendicitis but not effectively predicting perforation. Therefore, the diagnostic value of routine urinalysis may not appear high enough in clinical application. Secondly, repeated urinalysis examinations after surgery were not performed for surgical patients, therefore, cannot be entirely confident in ruling out other urinary disorders in these patients. ${ }^{1}$

\section{Conclusion}

Perforated appendicitis has a significant associated morbidity and mortality as compared to the non-perforated one. Measures to avoid the delay in reporting of patients at expert health care facilities should result in decreasing the incidence of these preventable complications. ${ }^{2}$ Routine urinalysis may reveal different findings between simple and perforated appendicitis. Clinically, we believe these urine parameters may aid primary emergency physicians in decision making for patients with suspected appendicitis. ${ }^{1}$

\section{Acknowledgements}

None.

\section{Conflict of interest}

The author declares no conflict of interest.

\section{References}

1. Wei CS, Wu HP, Chang YJ. Routine urinalysis in patients with acute appendicitis. J Emerg Crit Care Med. 2007;18:71-78.

2. Trautlein JJ, Lambert RL, Miller J. Malpractice in the emergency department-review of 200 cases. Ann Emerg Med. 1984;13(1):709-711.

3. Azez AK, Kazim TJ, Abdul-alkareem MF. Abnormal urinalysis in acute appendicitis. Diyala Journal of Medicine. 2014;7:60-65.

4. Andersson RE. Meta-analysis of the clinical and laboratory diagnosis of appendicitis. Br J Surg. 2004;91(1):28-37. 